\title{
Towards Provable Security of Substitution-Permutation Encryption Networks
}

\author{
Zhi-Guo Chen and Stafford E. Tavares \\ Department of Electrical and Computer Engineering \\ Queen's University at Kingston, Ontario, Canada, K7L 3N6 \\ \{chenz, tavares\}@ee.queensu.ca \\ http://adonis.ee.queensu.ca:8000
}

\begin{abstract}
This paper investigates some security properties of basic substitution-permutation encryption networks (SPNs) by studying the nonlinearity distribution and the XOR table distribution. Based on the idea that mixing small weak transformations results in a large strong cipher, we provide some evidence which shows that a basic SPN converges to a randomly generated s-box with the same dimensions as the SPN after enough number of rounds. We also present a new differential-like attack which is easy to implement and outperforms the classical differential cryptanalysis on the basic SPN structure. In particular, it is shown that 64 -bit SPNs with $8 \times 8$ s-boxes are resistant to our attack after 12 rounds. All of above effort may be regarded as the first step towards provable security for SPN cryptosystems.
\end{abstract}

Keywords: block cipher, nonlinearity, XOR table, differential attack, provable security.

\section{Introduction}

Substitution-permutation encryption networks (SPNs) were first suggested by Feistel 2 as a simple and effective implementation of private-key block ciphers (symmetric ciphers) based on the concept of "confusion" and "diffusion" introduced by Shannon 14. An SPN is constructed by a number of rounds of nonlinear substitutions (s-boxes) followed by bit permutations. Keying the network can be accomplished by XORing the key bits with the data bits before each round of substitutions and after the last round. The key bits associated with each round are derived from the master key according to the key scheduling algorithm. An example of a small SPN with $\mathrm{N}=16, \mathrm{n}=4$ and $\mathrm{R}=3$ is illustrated in Fig. II where $\mathrm{N}$ represents the block size of the SPN consisting of $\mathrm{R}$ rounds of $n \times n$ s-boxes.

There are two powerful classes of cryptanalytic attacks that can be mounted against block ciphers such as SPNs. Differential cryptanalysis II exploits a highly probable differential characteristic derived from the XOR table of the s-boxes II. Linear cryptanalysis $\varangle$ depends on the best linear approximation which is directly related to nonlinearity, an important cryptographic property. More details of these attacks on SPNs can be found in 6. On the other hand, it has been proved that completeness or nondegeneracy 7 can be achieved in the design of SPN cryptosystems. Avalanche characteristics are well studied in 5

S. Tavares and H. Meijer (Eds.): SAC'98, LNCS 1556, pp. 43- бо 1999.

(C) Springer-Verlag Berlin Heidelberg 1999 


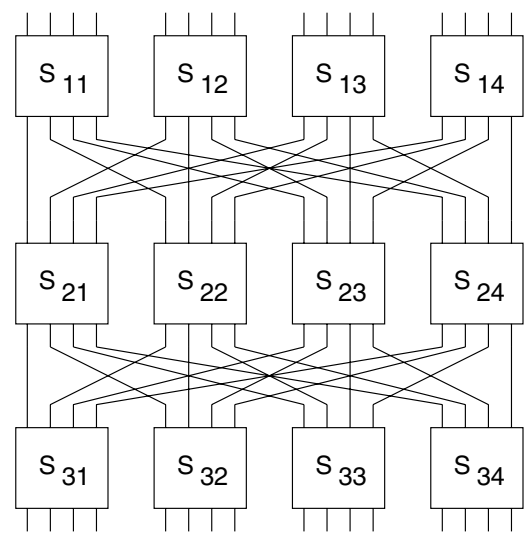

Fig. 1. $\mathrm{SPN}$ with $\mathrm{N}=16, \mathrm{n}=4$ and $\mathrm{R}=3$

and 18. All of the above suggests that the basic SPN has many desirable and predictable cryptographic properties useful for the design of cryptosystems.

In this paper, an estimate and upper bound on the nonlinearity distribution of bijective (invertible) s-boxes is presented. Based on the experimental results on nonlinearity and XOR table distribution, we show that the basic SPN resembles random bijective s-boxes of the same size with an increasing number of rounds, i.e., it converges to the ideal cipher. In addition, we present a practical differential-like attack on basic SPNs which exploits the Markov chain model based on the number of active s-boxes 18 . Our attack is effective regardless of the key-scheduling algorithm and more efficient than classical differential cryptanalysis. From the attack, we are able to find some hints on proving the security of SPN cryptosystems.

\section{Background}

\subsection{Nonlinearity}

A Boolean function $f(X)$ is an affine function if $f(X)=A \cdot X \oplus b$ where $X, A$ $\in\{0,1\}$, $b \in\{0,1\}$; ". " is the dot product and " $\oplus$ " is the XOR operation. Affine functions with $b=0$ are called linear functions. The set of all n-bit affine functions is denoted by $\mathcal{A}_{n}$. The set of all n-bit Boolean functions is denoted by $\mathcal{F}_{n}$.

The Hamming weight of a function $f \in \mathcal{F}_{n}$ is the number of ones in its truth table, denoted by $w t(f)$. The Hamming distance between two functions $f, g \in \mathcal{F}_{n}$ is defined as $w t(f \oplus g)$. A function $f \in \mathcal{F}_{n}$ with $w t(f)=2^{n-1}$ is said to be balanced.

We define the nonlinearity of a Boolean function $f \in \mathcal{F}_{n}$ as the minimum Hamming distance to all affine functions, denoted by

$$
N L(f)=\min _{g \in \mathcal{A}_{n}} w t(f \oplus g) .
$$


Let $\mathcal{O}_{n}$ denote the set of all non-zero linear combinations of output functions of an $n \times n$ s-box, i.e., $\mathcal{O}_{n}=\left\{f \mid f=a_{1} \cdot f_{1} \oplus \cdots \oplus a_{n} \cdot f_{n}\right\}$ where $a_{i} \in\{0,1\}$, all $a_{i} \neq 0$ and $f_{i}$ is the $i$-th output function of the s-box. Then the nonlinearity of the s-box is defined as the minimum nonlinearity of all functions in the set $\mathcal{O}_{n}$ :

$$
N L(S)=\min _{f \in \mathcal{O}_{n}} N L(f)
$$

\subsection{XOR Table}

A dynamic property of an s-box is the XOR difference table. For a given input difference, it provides possible input values to the s-box which generate the corresponding output difference. We define the entry of the XOR difference table for an $n \times n$ s-box with the input difference and the output difference $\Delta X, \Delta Y \in$ $\{0,1\}^{n}, \Delta X, \Delta Y \neq \mathbf{0}$ as follows:

$$
X O R(\Delta X, \Delta Y)=\left\{X \mid X \in\{0,1\}^{n}, S(X) \oplus S(X \oplus \Delta X)=\Delta Y\right\} .
$$

If each entry of the XOR distribution table is replaced by the number of elements in that entry, we consider the number as the XOR value and the new table as the XOR table. The largest value in the XOR table is called the XOR value of the s-box, denoted by $X O R^{*}$.

\section{Nonlinearity Distribution}

An output ciphertext bit of an SPN can be described as a nonlinear function of the input plaintext and the key bits. The nonlinearity of this function depends on the nonlinearity of the s-boxes, the only nonlinear components in the SPN cipher. If the nonlinearities of the s-boxes are very small, the cipher would be subject to a linear attack which makes use of a linear approximation to compute the key bits. Even if the nonlinearities of the s-boxes are made very high, it is still unknown whether the final SPN is highly nonlinear. However, Ritter I.3 uses experiments to show that the mixing constructions (permutations in the basic SPN structure) produce nonlinearity levels and distributions similar to those of an ideal cipher, i.e., a keyed look-up table of sufficient size. So what is the nonlinearity distribution? In this section we investigate the nonlinearity distribution of balanced Boolean functions and bijective s-boxes. (We also obtained similar results for random Boolean functions and s-boxes. We leave them out since they are not related to this work.)

Lemma 1. For a bijective s-box, all non-zero linear combinations of the output functions are balanced.

Lemma 2. The probability that the nonlinearity of a randomly selected $n$-bit balanced Boolean function $f$ is equal to $2 i$ is upper bounded by

$$
\operatorname{Pr}(N L(f)=2 i) \leq \frac{\left(2^{n+1}-2\right)}{\left(\begin{array}{c}
2^{n} \\
2^{n-1}
\end{array}\right)}\left(\begin{array}{c}
2^{n-1} \\
i
\end{array}\right)^{2}
$$

where "=" holds if $i<2^{n-3}$. 
Proof. The total number of balanced affine functions is $2^{n+1}-2$. For each of them, there are $\left(\begin{array}{c}2^{n-1} \\ i\end{array}\right)^{2}$ balanced functions at Hamming distance $2 i$. So the total number of balanced functions with nonlinearity $2 i$ is upper bounded by their product. For $i<2^{n-3}$, the bound is tight because these balanced functions are distinct. The result follows by noting that the total number of balanced functions is $\left(\begin{array}{c}2^{n} \\ 2^{n-1}\end{array}\right)$.

Theorem 3. For an $n \times n$ bijective $s$-box, the probability that its nonlinearity is less than or equal to $2 i$ is upper bounded by

$$
\operatorname{Pr}(N L(S) \leq 2 i) \leq \frac{2\left(2^{n}-1\right)^{2}}{\left(\begin{array}{c}
2^{n} \\
2^{n-1}
\end{array}\right)} \sum_{j=0}^{i}\left(\begin{array}{c}
2^{n-1} \\
j
\end{array}\right)^{2}
$$

Proof. Note that

$$
\operatorname{Pr}(N L(S) \leq 2 i) \leq\left(2^{n}-1\right) \operatorname{Pr}(N L(f) \leq 2 i)
$$

The upper bound in Lemma 2 can be used as an approximation to the nonlinearity distribution of a balanced function provided the nonlinearity is not very high. So if we assume that all non-zero linear combinations of all s-box output functions are independent in terms of nonlinearity, then we can get the approximation since

$$
\operatorname{Pr}(N L(S) \leq 2 i) \approx 1-(1-\operatorname{Pr}(N L(f) \leq 2 i))^{2^{n}-1}
$$

It can be seen that the approximation and upper bound are close in Table I We tested $10^{7}$ random bijective s-boxes of size $8 \times 8$. Only probabilities for nonlinearity from 80 to 98 are shown.

A more complete nonlinearity distribution is plotted in Fig. 2 according to the approximation expression $\mathbf{n}$. It indicates that s-boxes with nonlinearity greater than 98 are extremely rare. At the low end, the probability also decreases dramatically. So most s-boxes have nonlinearities between 80 and 98 . For example, $\operatorname{Pr}(N L(S)<80)=3.63 \times 10^{-5}$ and $\operatorname{Pr}(N L(S)>98)=1.01 \times 10^{-8}$. Linear s-boxes (whose nonlinearities are 0 ) are very unlikely to occur since the probability is about $2.26 \times 10^{-71}$ which agrees with the previous result in 3 . (After we allow for the fact that 3 uses an old definition of nonlinearity.)

\section{XOR Distribution Table}

The success of differential cryptanalysis relies on the existence of a highly probable differential which is equivalent to the existence of a large value in the XOR table of the SPN cipher, where we view the entire cipher as a big s-box. Unfortunately, it is impractical to examine the properties of the XOR tables of large 
Table 1. Experimental result versus theoretical approximation in $\mathbf{7}$ and upper bound in 5 for the nonlinearity of random bijective $8 \times 8$ s-boxes

\begin{tabular}{|c|c|c|c|}
\hline NL $\leq$ & Experiment & Theory & Bound \\
\hline \hline 80 & $1.83 \times 10^{-4}$ & $1.83 \times 10^{-4}$ & $1.83 \times 10^{-4}$ \\
\hline 82 & $8.70 \times 10^{-4}$ & $8.57 \times 10^{-4}$ & $8.57 \times 10^{-4}$ \\
\hline 84 & $3.76 \times 10^{-3}$ & $3.74 \times 10^{-3}$ & $3.75 \times 10^{-3}$ \\
\hline 86 & $1.52 \times 10^{-2}$ & $1.52 \times 10^{-2}$ & $1.53 \times 10^{-2}$ \\
\hline 88 & $5.68 \times 10^{-2}$ & $5.68 \times 10^{-2}$ & $5.85 \times 10^{-2}$ \\
\hline 90 & $1.88 \times 10^{-1}$ & $1.89 \times 10^{-1}$ & $2.09 \times 10^{-1}$ \\
\hline 92 & $5.03 \times 10^{-1}$ & $5.03 \times 10^{-1}$ & $6.99 \times 10^{-1}$ \\
\hline 94 & $8.89 \times 10^{-1}$ & $8.89 \times 10^{-1}$ & - \\
\hline 96 & $9.98 \times 10^{-1}$ & $9.99 \times 10^{-1}$ & - \\
\hline 98 & $1.00 \times 10^{-0}$ & $1.00 \times 10^{-0}$ & - \\
\hline
\end{tabular}

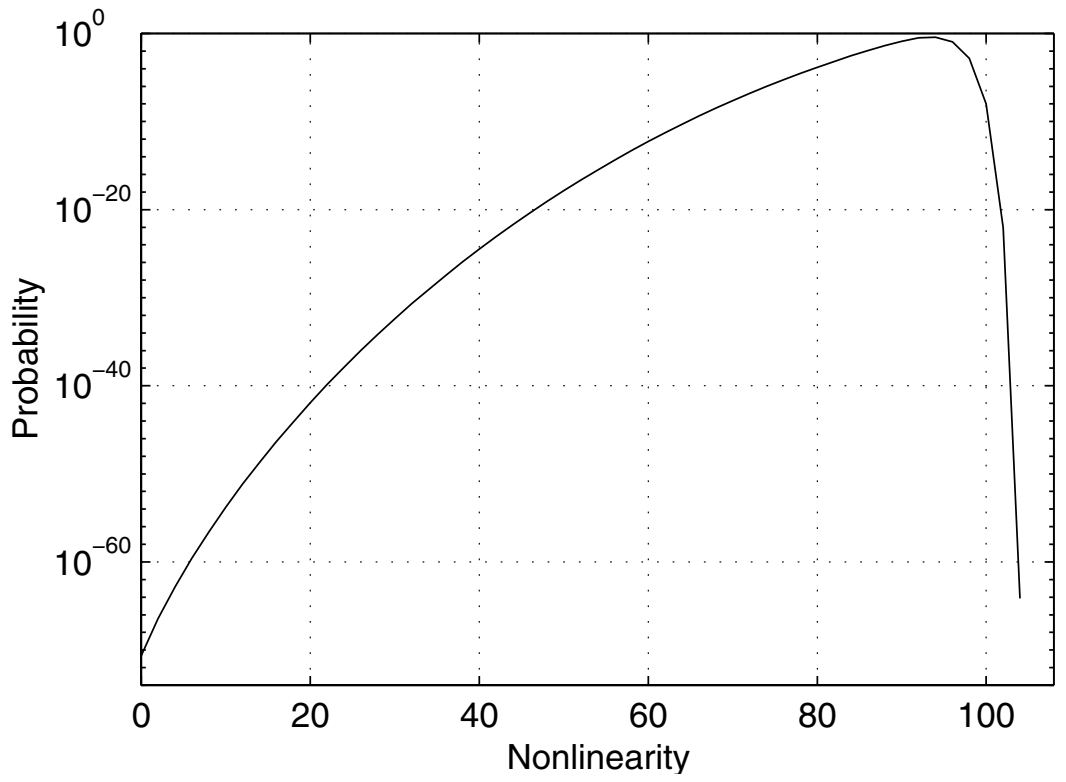

Fig. 2. Nonlinearity distribution for $8 \times 8$ s-boxes based on the approximation expression in 
SPNs (e.g., 64-bit SPNs). One advantage of the basic SPN is its simple "scalable" structure which make it possible to study a smaller version like the 16-bit SPN. Then we may intuitively extrapolate the results to 64-bit SPNs since they are constructed in a similar way. Thus, in this section we consider the 16-bit SPNs (see Fig. II and the corresponding $16 \times 16$ bijective s-boxes. We use the chi-square test to show that the XOR table of the basic SPN resembles a large random XOR table as the number of rounds increases. This statistical method is also used as a cryptanalytic attack in 4 and 16 .

\subsection{Chi-square Test}

The first observation is that there are many large entries in the first row of the XOR table of an SPN with a small number of rounds. Based on one experiment, we plot the frequencies of the entries in the first row for SPNs with 4, 5, 6, and 7 rounds, respectively, in Fig. $\mathbf{3}$

Although the plot is only one sample and cannot stand for the general case, the distinct differences of the entry distribution show that it is sensitive to the number of rounds. Furthermore, the entry distribution tends to stabilize with increasing number of rounds. It is natural to compare the XOR distribution table of an SPN to that of a random s-box (ideal cipher). We then employ the chi-square test to provide a quantitative measure of the difference.

Chi-square test is a standard test for the comparison of two distributions for binned data 12. The chi-square statistic is defined by

$$
\chi^{2}=\sum_{i} \frac{\left(R_{i}-S_{i}\right)^{2}}{R_{i}+S_{i}} .
$$

where both $R_{i}$ and $S_{i}$ are experimental data. Any term with $R_{i}=S_{i}=0$ is omitted from the sum. In general, a large value of $\chi^{2}$ indicates a large difference between the two distributions. The above method is described in 12.

\subsection{Comparison between SPNs and Random S-boxes}

Now we use the chi-square test 8 to examine the entry distribution in the XOR table of SPNs and random s-boxes. As an example, we compare 5-round and 8-round 16-bit SPNs to a random $16 \times 16 \mathrm{~s}$-box with respect to the first row of the XOR tables. We use the random s-box as the reference. The result is shown in Table $\boldsymbol{\nu}$ where the frequencies of the corresponding XOR values and the chi-square values are shown. The threshold for 6 degrees of freedom and $1 \%$ significance level is 16.81 . Therefore the 5 -round SPN is rejected and the 8-round SPN is accepted, which means that an 8-round SPN behaves like a random cipher while a 5-round SPN does not in this particular test. 

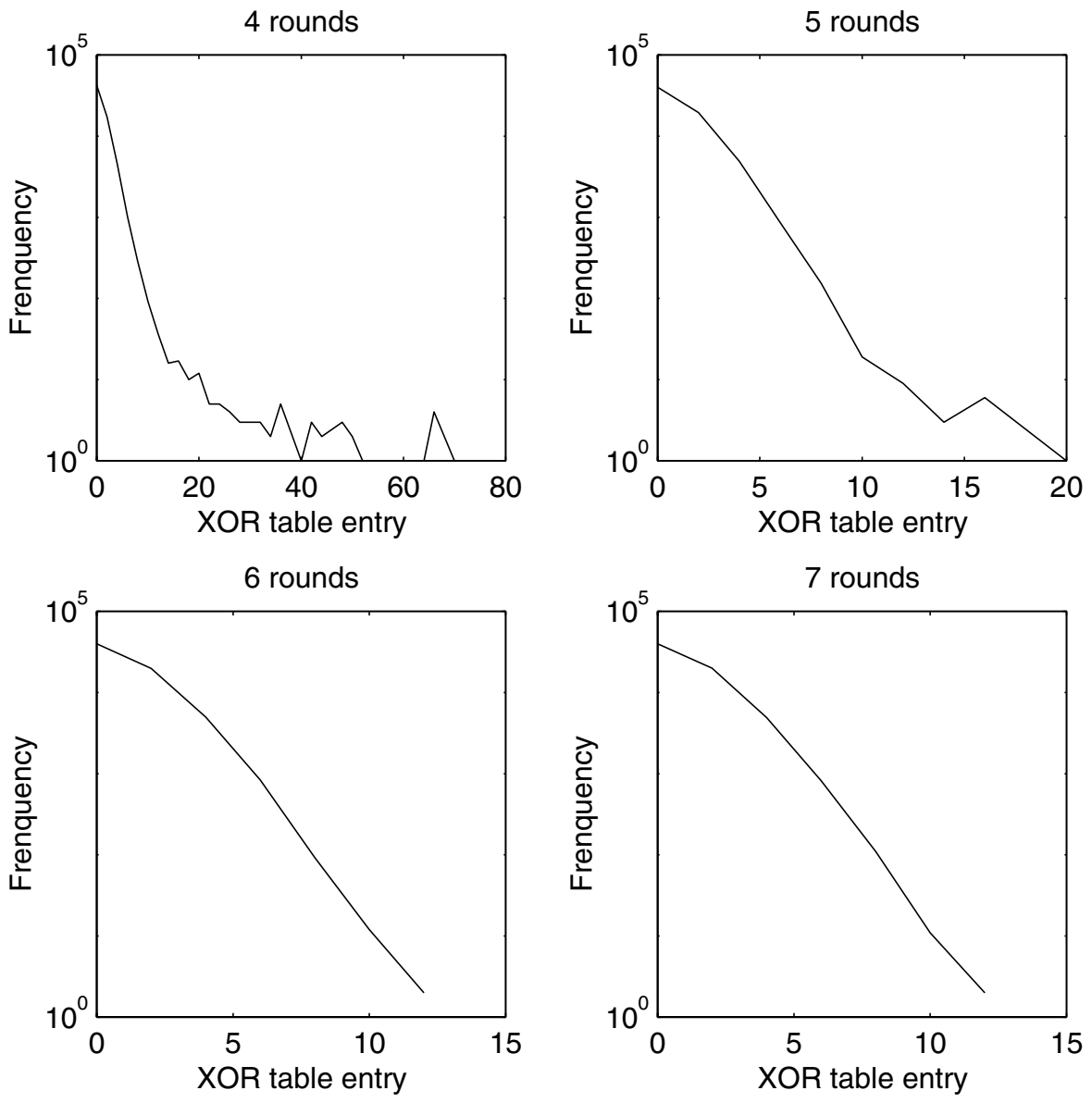

Fig. 3. The entry distribution in the first row of the XOR table for a "sample" $16 \times 16 \mathrm{SPN}$

Although small chi-square values do not always mean that the distribution is ideal (i.e., looks random), consistent large chi-square values indicate a significant difference. Thus for a complete comparison, it is straightforward to compare every row and consider the average of chi-square values. However, our experimental results show that the chi-square values are sensitive to the row index, i.e., the input difference $\Delta X$. It is found that for those input differences which influence a large number of s-boxes in the first round of the SPN, the corresponding row of the XOR table is "closer" to that of the random cipher than those input differences which influence a small number of s-boxes in the first round of the SPN. For example, Table 3 shows the chi-square values for the input difference of 0001, 0011, 0111, and 1111 (in hexadecimal format) respectively. 
Table 2. Chi-square test for SPNs and random s-boxes of size $16 \times 16$ (the threshold is 16.81)

\begin{tabular}{|c|c|c|c|}
\hline $\mathrm{XOR}=$ & random s-box & 5-round SPN & 8-round SPN \\
\hline \hline 0 & 39721 & 40053 & 39779 \\
\hline 2 & 19917 & 19521 & 19819 \\
\hline 4 & 4972 & 4908 & 5002 \\
\hline 6 & 809 & 861 & 814 \\
\hline 8 & 107 & 155 & 108 \\
\hline 10 & 8 & 19 & 13 \\
\hline$\geq 12$ & 2 & 19 & 1 \\
\hline \hline$\chi^{2}$ & - & 34.43 & 1.92 \\
\hline
\end{tabular}

Table 3. Chi-square test for different rows of XOR table

\begin{tabular}{|c|c|c|c|c|}
\hline Rounds & $\Delta X=0001$ & $\Delta X=0011$ & $\Delta X=0111$ & $\Delta X=1111$ \\
\hline 3 & $5.53 \times 10^{3}$ & $1.71 \times 10^{3}$ & $3.40 \times 10^{2}$ & $3.33 \times 10^{1}$ \\
\hline 4 & $9.32 \times 10^{2}$ & $1.31 \times 10^{1}$ & $7.39 \times 10^{0}$ & $7.23 \times 10^{0}$ \\
\hline 5 & $4.93 \times 10^{1}$ & $8.81 \times 10^{0}$ & $4.06 \times 10^{0}$ & $4.43 \times 10^{0}$ \\
\hline 6 & $6.46 \times 10^{0}$ & $4.20 \times 10^{0}$ & $6.25 \times 10^{0}$ & $4.03 \times 10^{0}$ \\
\hline 7 & $1.55 \times 10^{0}$ & $6.07 \times 10^{0}$ & $2.81 \times 10^{0}$ & $1.27 \times 10^{0}$ \\
\hline 8 & $4.90 \times 10^{\circ}$ & $2.16 \times 10^{\circ}$ & $2.14 \times 10^{0}$ & $7.79 \times 10^{0}$ \\
\hline
\end{tabular}

Therefore, we should concentrate on the average over those rows where the input difference influences only one s-box in the first round. There are in total $\left(\begin{array}{l}4 \\ 1\end{array}\right) 15=60$ such rows. Figure 4 illustrates the chi-square test results based on one experiment. The maximum and minimum chi-square values are also presented. The average chi-square value can be regarded as a measure of the distance to the ideal cipher. It can be seen that on average the distribution of the XOR table resembles that of the ideal cipher with increasing number of rounds. And for the 16-bit SPN, we need at least 7 rounds to make the distribution "random". In fact, we usually need more rounds due to the effect of fluctuations. For 7 or more rounds, the average chi-square value is very close to the result of comparing two random s-boxes.

\section{Differential-like Attack}

Differential cryptanalysis of SPNs is based on the best characteristic instead of the best differential I0 . Heys and Tavares 6 derived upper bounds on the most likely differential characteristic as a function of the maximum XOR value and the number of active s-boxes (i.e., the s-boxes whose inputs are changed in the process of encrypting two plaintexts).

In this section, we present a new differential-like attack on basic SPNs. By modeling the number of active s-boxes in the network using Markov chains 18 , 


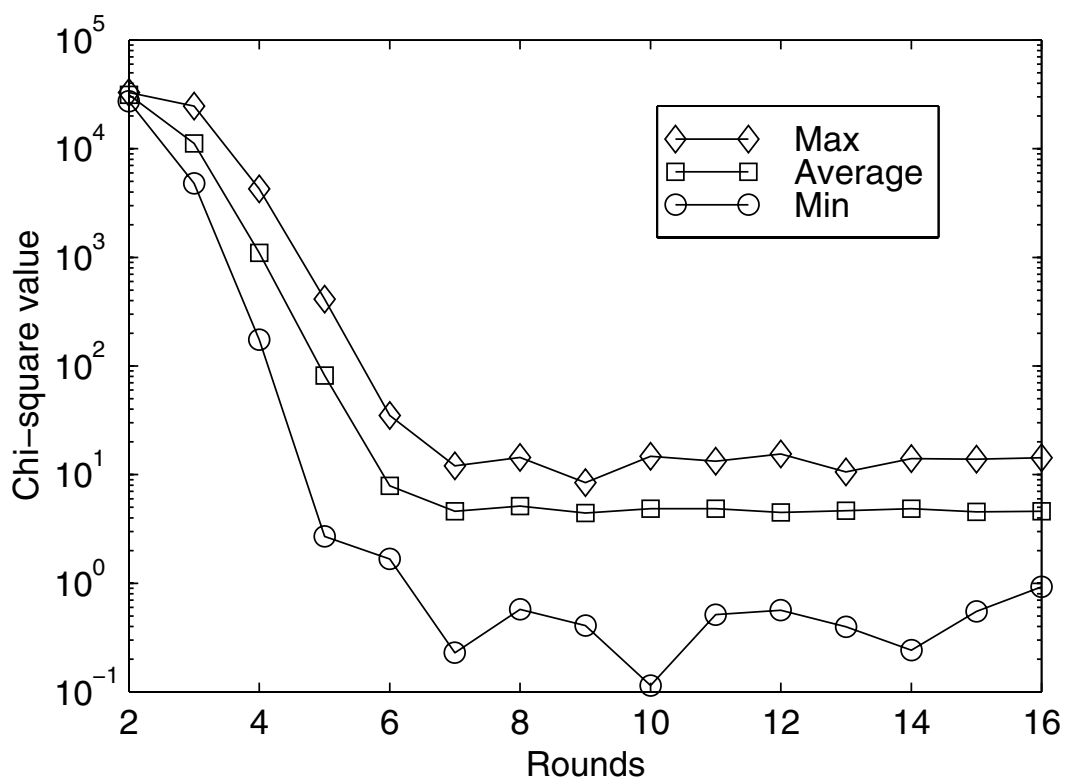

Fig. 4. Chi-square test for SPNs and random s-boxes with respect to the 60 rows of XOR table (with one active s-box in the first round)

we may predict the number of active s-boxes in the second round provided that we make one s-box in the first round (the target s-box) active and know the number of active s-boxes in the last round. This enables us to determine the subkeys of the first round and the subsequent rounds can be attacked similarly.

\subsection{Principle of the Attack}

Consider an $r$-round SPN with $n_{i}$ representing the number of active s-boxes in round $i(1 \leq i \leq r)$, the probability of $k$ active s-boxes in round $r$ given one active s-box in the first round is denoted by $\operatorname{Pr}\left(n_{r}=k \mid n_{1}=1\right)$. Actually, it is a transition probability of $r-1$ rounds. Now the selection matrix is defined by $\mathbf{S}=\left[s_{j k}^{(r)}\right]$ where

$$
s_{j k}^{(r)}=\frac{\operatorname{Pr}\left(n_{r}=k ; n_{2}=j \mid n_{1}=1\right)}{\operatorname{Pr}\left(n_{r}=k \mid n_{1}=1\right)} .
$$

In other words, $s_{j k}^{(r)}$ is the probability of having $j$ active s-boxes in the second round given that there is one active s-box in the first round and $k$ active sboxes in the last round. All of the above probabilities can be calculated from the transition matrix of Markov chains 18.

Now from the matrix $\mathbf{S}$ we may predict the number of active s-boxes in the second round by selecting those $s_{j k}^{(r)}$ greater than $50 \%$. If we know how many 
s-boxes are active in the second round, then we know the output changes of the target s-box. Since the exact inputs to the target s-box are known, we can increment the counters of possible subkeys according to the XOR table of the target s-box. After we examine a number of chosen plaintext pairs, the correct subkey will be counted more often than all the others. The same method is used to derive all subkeys in the first round. If the first round is broken, then we can break the subsequent rounds in the same manner.

We find that it is highly probable that only one s-box in the second round is active if a small number of s-boxes are active in the last round. This also conforms with our intuition. So only the first row of the matrix $\mathbf{S}$ is important. If we define the selection set of the $r$-round SPN as $\mathcal{T}_{r}=\left\{k \mid s_{1 k}^{(r)}>0.5\right\}$, then the algorithm for attacking the target s-box in the first round of the $r$-round SPN is:

1. Encrypt a pair of random plaintexts such that only the target s-box is active. If the number of active s-boxes in the last round is not in the set $\mathcal{T}_{r}$, then go to 1 .

2. Increment the counters of possible subkeys according to those XOR table entries which make only one s-box in the second round active. If there is no such subkey with counter greater than all the others by a threshold value (e.g., 2), then go to 1 .

3. Stop. The subkey of the target s-box is found.

The number of chosen plaintext pairs required to determine the subkeys in the first round may be approximated by $N_{P}=c / P_{d}$, where $c$ is a constant which may be approximated by $6 m$, and $m$ is the number of s-boxes in one round (similar to the results in 1 ), and

$$
P_{d}=\sum_{i \in \mathcal{T}_{r}} \operatorname{Pr}\left(n_{r}=i \mid n_{1}=1\right) .
$$

The threshold value corresponds to the confidence level of success. The higher the value, the more confidence we have that the subkey is correct and the more chosen plaintext pairs we need. The selection set may become empty for SPNs with increasing number of rounds, which suggests that they are immune to our attack.

\subsection{Experimental Results}

Our analytical results for the 64 -bit $\mathrm{SPN}$ with randomly selected $8 \times 8$ s-boxes are shown in Table 1 Here we define the complexity as the number of chosen plaintext pairs required to determine the first round subkeys according to the calculation of $N_{P}$ and a choice of $c=50$. Our attack is effective for up to 11-round SPNs. Moreover, if we guess the 8-bit subkey associated with the target s-box in the first round, we may attack one more round with complexity increased $2^{8}$ times. But this is not practical in that the required plaintext pairs for 12 
Table 4. Differential-like cryptanalysis of a 64 -bit SPN with $8 \times 8$ s-boxes

\begin{tabular}{|c|c|}
\hline Rounds & Complexity $\left(N_{P}\right)$ \\
\hline 2 & $2^{11}$ \\
\hline 3 & $2^{12}$ \\
\hline 4 & $2^{14}$ \\
\hline 5 & $2^{19}$ \\
\hline 6 & $2^{25}$ \\
\hline 7 & $2^{30}$ \\
\hline 8 & $2^{36}$ \\
\hline 9 & $2^{43}$ \\
\hline 10 & $2^{47}$ \\
\hline 11 & $2^{55}$ \\
\hline 12 & $2^{63}$ \\
\hline
\end{tabular}

rounds is approximately equal to the total number of plaintext pairs available for a 64 -bit SPN.

A simulation program was run to attack a 64-bit SPN composed of $8 \times 8$ sboxes with maximum XOR table entry $X O R^{*}=4$. The experimental results for up to 8 round 1 are plotted in Fig. 5 which also shows the theoretical complexity of our new attack and classical differential cryptanalysis. Note that the example seems unfavorable to differential cryptanalysis since we use a set of s-boxes with small $X O R^{*}$ which are generated from 9 and 17 . The expected $X O R^{*}$ value of a randomly selected $8 \times 8$ bijective s-box is upper bounded by $16 \mathrm{IL}$. But in practice, the value is about 12 and the highly probable characteristic can not always make use of all of these large values. So our attack outperforms the classical differential attack in a practical sense.

\subsection{Comments on the Attack}

There is an improvement in the implementation of our attack. By carefully choosing such plaintext pairs that make one s-box in the second round active more likely (this can be achieved by inspecting the XOR table of the target s-box), we may enhance the attack by a factor of two. However, the gain is not significant for attacking a large number of rounds.

In fact, our attack exploits the slow avalanche effect of basic SPNs. So the use of s-boxes with a high diffusion order 6 could minimize the impact of the attack. In addition, by replacing the permutation with the linear transformation $b$ or multipermutations I.5, we could also thwart the attack effectively. However, this introduces a delay which is significant for software implementation.

Both our attack and the classical differential attack are chosen plaintext attacks. The fundamental difference between our attack and the classical attack is that there is a filtering process in our attack.

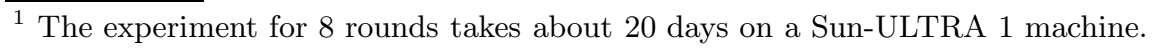




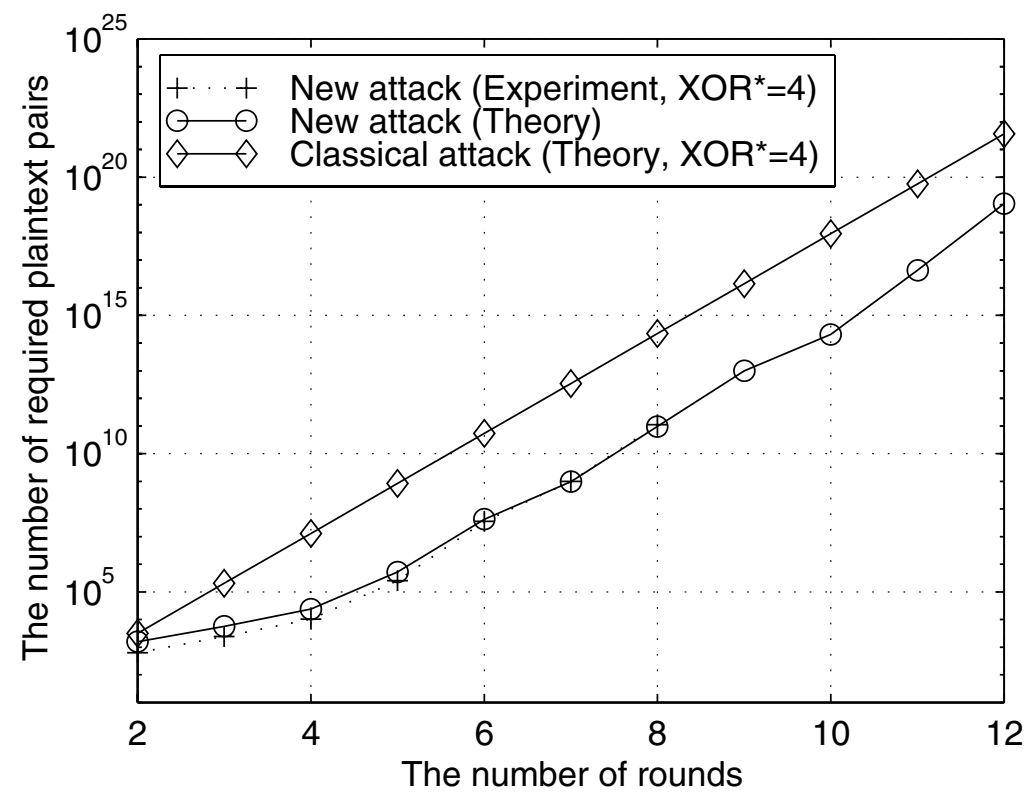

Fig. 5. Comparison of classical differential cryptanalysis and our new attack on a 64 -bit SPN with $8 \times 8$ s-boxes

Our attack first selects the ciphertexts, then checks those selected, while the classical attack checks every ciphertext trying to derive the key. Hence, our attack can easily take advantage of distributed computing (e.g., over the Internet). Furthermore, it is easy to implement our attack since only minimal preliminary analysis is needed.

In order to get secure SPNs, we need to make the SPNs behave like random big s-boxes. It is necessary to make the one-bit propagation probability less than the random probability so that the SPNs is not distinguishable from the random s-boxes. This results in an estimate of the minimum number of rounds required, denoted by $r$, as follows:

$$
\left(\frac{n}{2^{n}-1}\right)^{r} \leq \frac{n^{2}}{2^{n^{2}}-1} .
$$

where $n$ is the size of s-boxes. After simplifying the above expression, we can get

$$
r>n+\log _{2}(n) .
$$

Then it can be seen that when $n=4$ (i.e., for 16 -bit SPNs), we need at least 7 rounds, and when $n=8$ (i.e., for 64-bit SPNs), we need at least 12 rounds. These agree with our previous results. 


\section{Conclusion}

We have presented an upper bound on the nonlinearity distribution of randomly selected bijective s-boxes which shows that low nonlinearities are very unlikely for large s-boxes. Note that we have only considered bijective s-boxes throughout this work. An SPN may be regarded as a set of large s-boxes indexed by the keys.

Based on the experimental results on XOR table distributions and supported by the results on nonlinearity 13, we have shown that the basic SPN converges to the ideal cipher with an increasing number of rounds. In addition, we have presented a practical differential-like attack on basic SPNs. From the attack, it can be seen that the number of active s-boxes is very important. For a secure $\mathrm{SPN}$, it is necessary to make the number of active s-boxes in the last round independent of the number of active s-boxes in previous rounds. This may be equivalent to $\operatorname{Pr}(\Delta Y \mid \Delta X)=\operatorname{Pr}(\Delta Y)$ (where $\Delta X$ and $\Delta Y$ are plaintext and ciphertext differences respectively) which implies that the ciphertext is a random permutation of plaintext. Based on Markov chains 18, it is found that the number of active s-boxes in the last rounds tends to be independent for basic SPNs with an increasing number of rounds. These experiments and analytical estimates may be regarded as some evidence towards provable security for SPN cryptosystems.

\section{References}

1. E. Biham and A. Shamir. Differential cryptanalysis of DES-like cryptosystems. Journal of Cryptology, vol. 4, no. 1, pp. 3-72, 1991

2. H. Feistel. Cryptography and computer privacy. Scientific American, vol. 228, pp. 15-23, 1973

3. J. Gordon and H. Retkin. Are big S-Boxes Best? Proc. of the Workshop on Cryptography, LNCS 149, Berlin, pp. 257-262, 1982

4. H. Handschuh and H. Gilbert. $\chi^{2}$ Cryptanalysis of the SEAL Encryption Algorithm. Fast Software Encryption, LNCS 1267, Springer-Verlag, pp. 1-12, 1997

5. H.M. Heys and S.E. Tavares. Avalanche characteristics of substitution-permutation networks. IEEE Trans. Comp., vol. 44, pp. 1131-1139, Sept. 1995

6. H.M. Heys and S.E. Tavares. Substitution Permutation Networks Resistant to Differential and Linear Cryptanalysis. Journal of Cryptology, vol. 9, no. 1, pp. 1-19, 1996

7. J.B. Kam and G.I. Davida. Structured design of substitution-permutation encryption networks. IEEE Trans. Comp., C-28, pp. 747-753, 1979

8. M. Matsui. Linear cryptanalysis method for DES cipher. Proc. of Eurocrypt '93, Springer-Verlag, Berlin, pp. 386-397, 1994

9. K. Nyberg. Differentially uniform mappings for cryptography. Advances in Cryptology: Proc. of EUROCRYPT '93, Springer-Verlag, Berling, pp. 55-64, 1994

10. K. Nyberg and L.R. Knudsen. Provable security against differential cryptanalysis. Advances in Cryptology: Proc. of CRYPTO '92, Springer-Verlag, pp. 566-574, 1993

11. L.J. O'Connor. On the distribution of characteristics in bijective mappings. Proc. of Eurocrypt '93, Springer-Verlag, Berlin, pp. 360-370, 1994

12. W.H. Press, B.P. Flannery, S.A. Teukplsky and W.T. Vetterling. Numerical Recipes in $C$ - The Art of Scientific Computing, Cambridge University Press, pp. 487-494, 1988 
13. T. Ritter. Measured nonlinearity in mixing constructions. http://www.io.com/ ritter/

14. C.E. Shannon. Communication theory of secrecy systems. Bell System Technical Journal, Vol. 28, pp. 656-715, 1949

15. S. Vaudenay. On the need for multipermutations: cryptanalysis of MD4 and SAFER. Fast Software Encryption, LNCS 1008, Springer-Verlag, pp. 286-297, 1995

16. S. Vaudenay. An Experiment on DES - Statistical Cryptanalysis. Proc. of the 3rd ACM Conference on Computer Security, ACM Press, pp. 139-147, 1996

17. A.M. Youssef, Z.G. Chen, and S.E. Tavares. Construction of highly nonlinear injective s-boxes with application to CAST-like encryption algorithms. Proc. of the Canadian Conference on Electrical and Computer Engineering (CCECE '97), pp. 330-333, 1997

18. A.M. Youssef. Analysis and Design of Block Ciphers. PhD thesis, Queen's University, Kingston, Canada, 1997 